\title{
The Analysis on the Techniques and Skills of the Action Set in the Three-Dimensional Animation Manufacture
}

\author{
Xiao Zhang ${ }^{1, \text { a }}$ \\ ${ }^{1}$ Movie and Media College, Sichuan Normal University, Chengdu, 610068, China \\ a15982182811@163.com
}

Keywords: Three-Dimensional Animation, Action Set, Techniques, Skills

\begin{abstract}
With the rapid development of information, 3D animation technology has become the indispensable technical means in the field of video effects, education and scientific research. The three-dimensional animation is a comprehensive art. The action set has been the key step in a three-dimensional animation. Among them, a good action movie set is the foundation to improve the quality of animation and can help animators improve the efficiency of completing three-dimensional animation. This paper analyzes the techniques and skills of action movie set in three-dimensional animation.
\end{abstract}

\section{Introduction}

The nature of three-dimensional computer animation computer graphics and art of combining the product is accompanied by a high-tech computer technology developed. With the information society covering an increasingly wide range of computer information, three-dimensional computer animation businesses also played a growing role. Three-dimensional computer animation technology is used in film special effects, animation, large-scale game production, military flight simulation, simulation exercises case even for analog reproduction. From this, we can see the three-dimensional computer animation technology showcase of powerful features. In three-dimensional computer animation technology, we can find the character animation technology and the role of action three-dimensional computer animation technology is the most shining place. In real life, for we can have an enormous influence and shocking undoubtedly use a lot of three-dimensional animation technology, television dramas and movies, such as "Transformers," "Iron Man," "Madagascar," "Shrek," "Lord of the Rings "Wait. The use of three-dimensional animation art digital video film shows us the characters of realism, gorgeous sense. Each film has a highly personalized charm of the unique role and the role of the iconic action, deep infection to set planning is very important [1].

\section{The Current Development of Computer Animation}

Throughout the history of the development of Chinese animation, two-dimensional animation can be found in China during the initial stage of development and foreign animation is almost comparable. China's first movie cartoon "row studio" in the early 1920s issued only Bidisini's "Steamboat Willie," launched two years later. After the 1990s, the traditional two-dimensional animation turned to three-dimensional computer animation. 1990 Eleventh Asian Games in Beijing, China Central Television, Beijing Television was televised for the first time in three-dimensional computer animation technology used to make television titles. Since then, computer animation technology began rapid development in China. However, compared with the level of international animation, computer animation industry in our country is only just beginning. Weak technological base, lack of funds, the overall level is relatively backward, lack of professional education influenced by several factors talents even restricted the development of three-dimensional animation. 


\section{The Action Set in the Three-Dimensional Animation Manufacture}

Three-dimensional animation in the role of action are basically three-dimensional software for manual adjustment. Use objects to fully consider the law of motion, the role of action may reflect the role of emotional changes. We make character action plan, to fully consider the role of the character. Reasonable, smoothness of operation in order to make the animated character truly "alive" in order to highlight the role of "vitality." Such as "Madagascar" in the lion, zebra and other roles, with their different personality action. Former good character action plan, we have to carefully observe the life, experience life, get more action inspiration from life, to understand the basic movement of the basic operation. As the saying goes, "remain the same." On the basis of our fundamental law, to change, and vividly demonstrates the role of personality [2].

\section{The Production Skills of Animation}

Animation production process can be divided into modeling, animation, rendering, special treatment of four parts, the use of software Maya, Rhino, Afterteffect, Morph, Premiere and so on.

Modeling. The basic structure can be built in a crude model Maya most turbine runner, and then with Loft, Booleans, Lattice and other ways to accomplish the work of fine model. If the model is too large can use Maya model comes with optimization program optimization. As Kaplan turbine runner chamber production: first reference data to determine the proportion of the size of the real model, draw on its three-view drawings, swept by the scanner into the computer, in Maya people will sweep pattern as background then fine modeling. Do runner chamber, the first model to do exactly the same entity with which the empty part of the volume, which is aligned with the center of gravity position and the runner chamber, eight times Boolean after the operation, to obtain the required basic model, based on this model use Editmesh extra points and adjust Boolean left to sleek, sophisticated modeling complete [3].

Special model with Rhino Nurbs powerful modeling, and then guide people Maya rendered, such as the production of spiral: As the spiral case of function and consistent water pressure turns into each vane, so it is respective sectional all different. In Maya with Loft is difficult to accomplish, while adjusting the line in Rhino point of flexibility, in favor of the production, the most critical is that it is very good sleek, volute produce lifelike image. Runner is in the water flow, only rotating turbine runner, runner blades without water impact, so the apparent lack of authenticity animation, is not conducive to teaching. How to show the flow of it? Particle system is the best tool for the performance of the water, but taking into account the water when people interact blade runner chamber, there is a rebound, gravity, and other physical phenomena lasing on the performance requirements of the water, if at all the leaves have shown, but see how it works, it did not make all of the water, but the performance of its work with part-flow principle.

Animation. During animation to rotate the guide vane linkage mechanism is the most complicated. Maya animation key frames set, manual setting automatic interpolation than good, because the model is automatically interpolated curve is asymptote mode, easy to make the animation deformation, especially small part of the animation, so the manual is to ensure the animation key framing quality of the premise. Although the choice of manual settings too much trouble, heavy workload, but high accuracy, the link with the vanes in this part of the animation on each vane 20 is set a few key frames, and set graph editor's curve regulator, has reached animation requirements, and more effective than automatic key frame animation is much better. In the rotational speed of the animation or movement, you should see the original structure, the rotational direction prevail; order parts removal and installation process should be consistent with the actual direction of rotation of the wheel to break and the actual operating conditions.

Render. Central parts of all animated color with white or black to form a larger contrast with the color of production, are made of black background, so there are two advantages, one can save storage space on your computer; the second is to improve the contrast, aims to improve the visual attention, enhance teaching effectiveness. 
On material we set the selection of a lot of metallic and concrete color, as to the performance of realism. But sometimes in order to more clearly show the different structure of the original, but with a variety of materials deliberately rendering an object of various parts, such as Tubular runner's performance on the use of the material to the performance of the five, but actually steel itself is only one color [4].

Rendering, in order to keep the number of lines and the high-resolution, all with 768x576 32-bit true color, although large files, but each produced an animation, immediately transferred to the non-linear as PAL-D system is recorded on the video tape, after clearing the original file, so 20G hard drive can flow, generally 20 seconds of animation rendering production takes about 6 hours to output, in particular the rebound and lasing performance particle system also requires a computer with high processing speed.

The Special Treatment. Maya used in the video a Post in the Glow filters, etc., in the expression of the various components of turbine structure with the most, when we want to elaborate runner when one component in the overall decomposition of the components in the first to make it made about light to indicate outstanding. After setting in video Post Glow filter, using a list in Traek modulating method can be achieved within one second to make a component to form a halo of flashing 3-5 down to play a prominent role.

To highlight the expression of three-dimensional animation, we use six different types of turbine runner plastic deformation. When more than one runner due to deformation by Maya process is too large, so using morPh2.5 as deformation software, it is not a model-based, but simply as the role of the object in the image, we can morph in each of the various types of put together the kind of runner, next to each pixel position of first occurrence of distribution, such as myself feel runner deformation. Final output uncompressed comes with Morph renderer TGA file sequence, which would achieve the desired deformation effect.

\section{Action Settings of the Role's Complex Behavior}

The Introduction of the Scene Editor. In the State 863 Project "public technology platform network game key technology research", the author's team eventually finished a combined graphics engine, physics engine, audio engine, AI, animation and other fabric modules scene editor system. The editor of the engine as a variety of high-level open-source engine and it can achieve multi-channel large visual real-time simulation of large-scale outdoor scenes.

The Basic Animation Mixing Experiments. In the Scene Editor, this module is mainly responsible for the following functions: forming a moving animation and avatar to match the actual velocity; smooth handover process between the two animation; implement action superimposed; providing a site for a role toward an object to maintain.

The Simple Human Physical Model Experiments. In practice, the project proposes a combination of physical model character animation motivation is to enhance the performance of force in crowd behavior module. In the editor, we selected OpenSteer as the controller of group behavior. Our aim is to use a simple physical model to simulate the character's walking behavior, to move it in the role of OpenSteer force and produce some irregular movement path of the disturbance and to eliminate OpenSteer force caused by sudden changes in direction untrue Role swerving situation.

The Complex Behavioral Experiments of Tiger Chasing A Deer. Experiments, the first to use the aforementioned animation hybrid technology to smooth handover process between the tiger and deer animation, animated tiger behavior include: resting, walking, running, lap, deer animations include: resting, walking, running. Tiger and deer are attached a simple physical model mentioned in the previous section, to enhance the realism of their movement. The last top control module is used to control the specific status of tiger and deer, which includes a OpenSteer group behavior module, used to maintain deer herd behavior; and the tiger's navigation control module, used to indicate the goal of the tiger; finally, a monitoring module distance between the Tigers and the target for the tiger responsible for the operation of the switching and deer [5]. 
The Basic Animation Mixing Experiments. Compared with a single animation sequence, animation mixing techniques has the following advantages. Animation can produce a large number of mixed use is difficult to adjust the action out directly through the insertion of two or more keyframe animation, can generate endless unique position. Actions such as waving join in walking animation. In the animation controllers, there are several tracks, under normal circumstances player animations are animated sequence on the first track, which is on track to play. Use animation hybrid approach is different paragraphs animation sequences are bound to different tracks, and then enable them to mix animation controllers will play out, thereby completing the animation mixed results.

\section{Conclusions}

Outstanding animated character should have full image and emotional actions. The action setting in three-dimensional animation is a very complex project, which requires cooperation with animators and all those staff who involved in the film production. In this article, the author discusses the techniques and skills of action set in the three-dimensional animation. The action set in three-dimensional animation is the basis for vivid film role and it has a very important role. We should thoroughly study the action settings of various roles in the three-dimensional animation to create a more vivid three-dimensional animation which has a distinct personality and deeply rooted for the audience.

\section{References}

[1] Xiaoding Ge: Journal of Shan Dong University, Vol. 6 (2004) No 53, p.25-26

[2] Xia Cai: Shaanxi Science and Technology University, Vol. 12 (2005) No 27, p.74-76

[3] Lujing Gao: Beijing industry university, Vol. 1 (2006) No 33, p.11-14

[4] Lijun Wan: Guangxi Normal University, Vol. 3 (2007) No33, p.121-124

[5] Bo Xia: Education Article, Vol. 15 (2012) No 28, p.178-181 